\title{
Using software such as "kuaishou" to shooting videos
}

\author{
Di Gu${ }^{1}$, Li Zhang ${ }^{2, *}$ \\ ${ }^{1}$ School of Media, Tonghua Normal University, Yucai Street, Tonghua, China \\ ${ }^{2}$ School of Physics, Tonghua Normal University, Yucai Street, Tonghua, China
}

\begin{abstract}
In a small stream line under the big trend, this paper gives a brief introduction to using software such as "kuaishou" to shooting a small video. This research concerns several parts: introduction, the writing of the script, the selection of actor, props, post-production and innovation. A small video is been made of five steps, based on this, it is been detailed on each piece of analysis to better complete the video. The purpose of this study is to show the methods mentioned above are promising. In this Internet age, this kind of video with one of the biggest bright spots, that is its powerful ability to turn into cash. The developed technique is applied to shooting videos for making money.
\end{abstract}

\section{INTRODUCTION}

In today's information age, a variety of small videos are very popular. In the present since the era of media, video has become one of the best marketing. However,those who really understand small video productions are few and far between. So, when learn a small video full set of production technology, there had the winning magic weapon. So how to make a nice little video?

\section{THE WRITING OF THE SCRIPT}

First of all, a good video must have a good script, the script writing has some basic requirements: scenario to bold, point to bold, left the scene of sound mark appear for the first time to be bold character name are centered bold character dialogue is in the middle and both sides left blank, different characters, start a new line of dialogue to show the relationship between the camera indicate the scene of switching, "cut" is hard to cut, the effect of "to" is to add transitions, all right, if you have special effects apply, also want to use boldface mark. These requirements not only can make according to this looks more neat and beautiful, can also is a clear understanding of the script, actor as soon as possible into the plot.

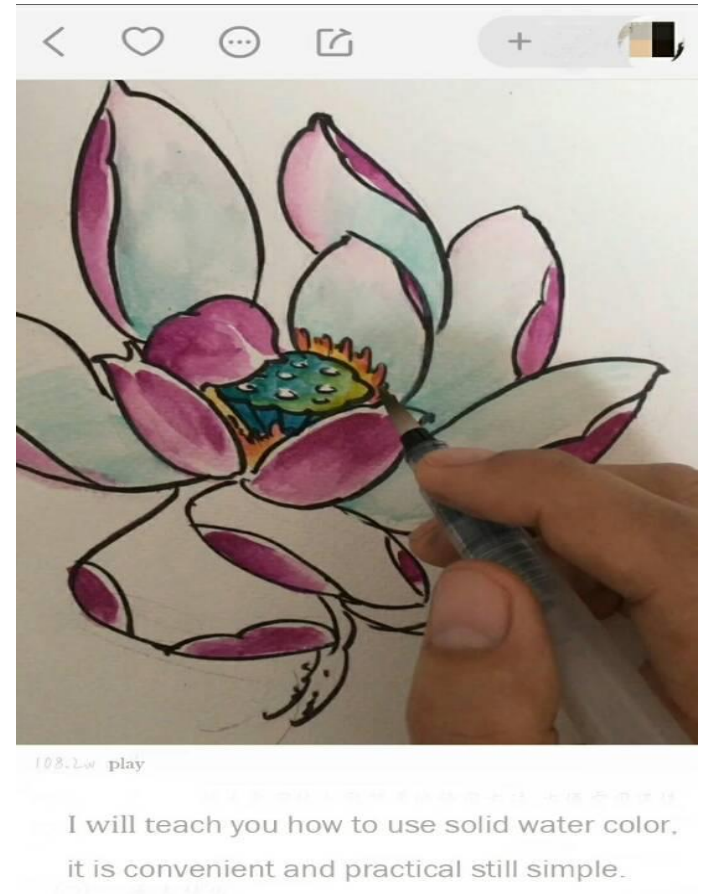

Figure 1: Video for teaching.

These are basic things, the most important thing is that content, script content must be innovative, positive upward, with positive energy. We can not imprisoned by inertial thinking, not to write some thinking, we can blend in education to the video, and everyone know the ending of love drama, divergent digital, networked, open will become the important direction of the construction of the teaching video resources Students are educated in batches, according to age, as if the most important thing they have in common. So our video according to the students in terms of the different stage. For example a fable story into a one of the five minutes of play, will learn knowledge express or explain or deductive way, and we can make some little about the food, beauty make

\footnotetext{
*Corresponding author: dbcy9999@163.com
} 
up programmes or funny video, and so on.As long as there is innovation, there will have a market.

\section{THE SELECTION OF ACTORS}

Selection of actors, this item is to be based on specific content to decide. If we want to select, the actor playing small plot so choose actors must accord with character requirements, its image, temperament and character requirements best. Imaging a temperament is good at the same time, to detect the mirror, it take a look at the actors in front of the camera's status as a whole is what kind of effect. Simple trying the actor's acting is in line with the requirements.

Such down sketch actor is chosen. In choosing the interpretation of education, food, beauty makeup person must be selected with professional quality or better at the people in the field.

\section{PROPS}

We hope you find the information in this template useful in the preparation of your submission.

When it comes to the selection of props, because we want to reduce costs to maximize profits, we should choose both economic and affordable. As some tables, chairs, blackboard, etc. These things have in our school, completely can be used on the promise of no damage to borrow, such as some decorations like little lights, goodlooking antependiums, background plates, etc.These we can choose appropriate to take out on the Internet. Props to this part of the effort and cost don't need too much.

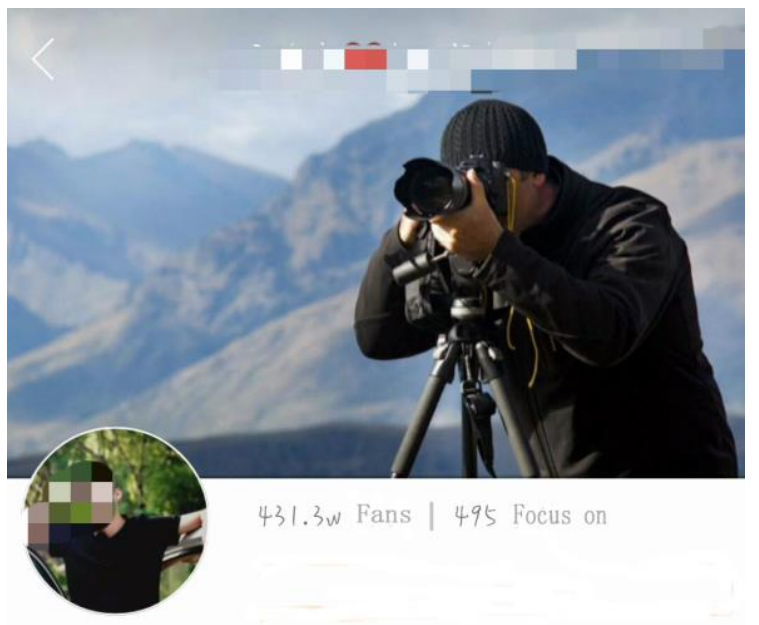

Figure 2: The focus of videos.

We want to focus attention is a video in the late film and video clips.

\section{THE VIDEO SHOOTING}

The development of photographic and film technology and the events which led to the rapid development of visual culture also point to the inseparable duality of observing visual anthropology within reactive and proactive processes, which are indirectly closely related to the technical aspects of these processes. Visual effects are at the forefront of a digital revolution in the film and video industry and are becoming more and more important to movie language. So what is the specific video shooting are required?

\subsection{POINT}

If it is outdoor scenes, can choose local representative landscape architecture; If it is outside, the best is the background and main body has a certain relationship, but not too mixed and disorderly or a presumptuous guest usurps the host's role. Sites it is best not to have too much noise (such as wind, the voice of a irrelevant personnel, ringtones, etc.), do not affect the scene of the shooting (if inevitable, as far as possible the guarantor's voice can hear).

\subsection{THE LIGHT}

As far as possible in both indoor and outdoor, give priority to with natural light, but avoid the scene very strong sunshine; In addition to creative needs, try to adopt suitable light or metering, avoid to use light and the light.

\subsection{PICTURE COMPOSITION}

Angle: in addition to the creativity needs to avoid shoot or Angle; Characters proportional position: reference golden section method, avoid too small or full of the whole picture; False or true: can be used long focal length, large aperture, small depth of field; Close blur of filming techniques such as the background, highlight the main body.

\subsection{THE BOOTH}

Control the panoramic, the middle (characters) knee above, close shot above the chest (figure) and the relationship between the features (face).

\subsection{INNOVATIVE}

We can bold innovation, best can be impressive, you'll never forget, leave a unique, unique impression.

\subsection{EQUIPMENT}

Use high quality cameras as far as possible, because now mobile phone software function of the shooting, and pixel can also, so you can also use the cell phone, but whether the use of mobile phones or the camera to shoot, shoot in the process of the fixed must be equipped with a tripod and other auxiliary equipment, has reached a stable effect. 


\subsection{TIME}

Because our shooting is a short video, so try to control our time in about five minutes, neither makes the audience feel boring, also can increase the audience's affection. Can apply these skills in photography, and when necessary article also can break a few boxes to achieve more perfect results.

\section{THE VIDEO POST-PRODUCTION}

Finally in making video clips, video clips of the postproduction is as important as pre-production. The advent of computer-generated imagery and digital effects has changed all that. Now anyone can have accessed to the technology used to create Jurassic Park, regardless of whether they are based in Los Angeles, in the South Pacific, on a Greek Island or in Brussels. We can draw lessons from the production of the film, the film postproduction process, it is this at the end of the film to edit operation. Both animation and video material in the initial production has been completed, are in the form of a single fragment exist individually, each fragment after splicing or special effects and many other work, to create a complete work. Paying attention to the correct use of appropriate software and the traditional linear editing and nonlinear editing, integrated use of the right in the later editing software can get twice the result with half the effort and aesthetic effect. Would be late edit video clips, must according to the production requirements of video clips and the characteristics of two kinds of edit mode, choose the right means of software. In the process of production, we want to keep the picture clean, first of all is to no clamp frame, is a complete lens links, no superfluous things. When we cut again if you have the camera at the end of the not cut, the back with a camera, but these things are due to mistakes with, we don't need, this is the clip frames. And when we connect the two lenses, no connection between good and black field, it is also a clip frames. Usually when editing, an important task is to cut the screen clean. And then there is the integrity of the lens, lens in editing mode by static/dynamic static dynamic in two ways. The last is the lens finally must be fixed, move the camera images cannot be ended, so the clip point moving lens must be quieted down at last, dynamic, even using the last scene must calm down. The main body to stop movement, or painting. Editing process we need to pay attention to the clean sound, first to complete, whether the original sound or narrator, not let people say half sentence. There is sound to pure, can not use the special noise, must let the audience understand it. The volume of the sound height to fit, a small video whole volume to be unified, cannot have high low. Background music volume proportion to the right, can't let the voice of the background music affect the voice.

On the effect of processing 1, transformation occurs in the scene, if from a scene, immediately turn to another scene, make you feel the picture is not coherent. So we need to add a process of transition, switch to the, this is transitions. A switch at the end of the video slowly from the former to the latter period of the beginning of the video, so you can feel the picture is a natural transition of 2 , in addition to the turn off we can also provide another effect of the number of video, that is a filter. Filter and transitions, he this video effect processing, the whole effect is accompanied by the whole video.3, we can add subtitles in the video,which can be explained in the whole added to the beginning of the video, or add a section in the middle of the show, so that we can help the audience to understand.

\section{THE INNOVATION}

So how you can let us work in numerous small video to stand out, this would require the two words: innovation. Through the application of creative capacity a vital dimension of human intelligence is demonstrated. Script to innovation, the performances to innovation, process innovation, production innovation. In this era of information updates quickly, we must use our wisdom and knowledge to innovate videos, we have studied and more refined processing and processing, to make our video quality be more good, can let the audience see from the many videos and remember our video, can also be added in the video more creative cover to attract viewers and thus increase our clicks and fans, click as well as the fans the more you will earn more money. We need to use all sorts of small videos now better prospects for development, and our professional knowledge, create more and better video.

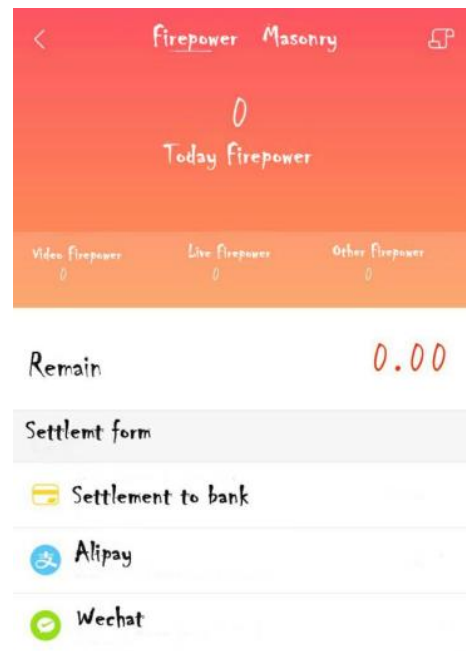

Figure 3: Profit in "Huoshan".

At the same time it also has considerable the ability of into cash, cash is the most important way of cash short video platform, in a platform on the video, with earnings platform to earn money by video playback volume share out bonus, and exceptional fans bonuses, platform, also channels can reap the benefits. Electricity to liquidate, do ing your own electricity uses short video drainage or uses taobao guest form channels which are good electricity can be sold and turned into money.

Advertisements, in the video broadcast data after stability, can meet the commercial short video or commercials for commission. Knowledge cash, needs to 
be done fans accumulation stage, and then do the paid knowledge output.So our topic is with great potential.

\section{CONCLUSION}

In the content of the above, this paper introduces the small video shooting process, its essence and other video shootings are consistent, taking only small video phone software can be used to shooting, so not as good as professional with a camera, but is enough for a small video, but also greatly reduced costs. For a small video, can be in detail and originality, because the video is only about five minutes, the idea of the script is very important.If you want to let the audience see at a first glance you are interested in, and can see the whole video, this scenario is to make great efforts one time. That's how you get the most benefit.

\section{ACKNOWLEDGEMENTS}

This work was supported by the College Student Project of Tonghua Normal University.

\section{REFERENCES}

1. Ben Stassen. 1997,31(1),Location-based entertainment-small is beautiful! ACM SIGGRAPH Computer Graphics.

2. Ioan Susnea Emilia Pecheanu, Cornelia Tudorie2015,. Procedia - Social and Behavioral Sciences.

3. Roy Prentice. Creativity , 2000,11(2) , a reaffirmation of its place in early childhood education, Curriculum Journal.

4. Niksa Svilicic,Zlatko Vidackovic.,2013,37(1).Film and Television in Croatia Today: Production, New Technologies and the Relationship with Visual Anthropology, Collegium Antropologicum.

5. Mitch Mitchell, Taylor and Francis 2013-07-31 Visual Effects for Film and Television.

6. Zhang wei. 2012, The development and application of network teaching video resources research, Liaoning normal university.

7. JPan Hulin. 2009 (15), The modern film and television post-production technology, computer knowledge and technology. 